\title{
Hysteroscopic morcelation of large type II myoma
}

\author{
Karin Abbink $^{1}$ (D) - Sameer Sendy ${ }^{2}$ Tawfig H. Gaafar ${ }^{3} \cdot$ Dick C. Schoot $^{1}$
}

Received: 23 March 2015 / Accepted: 2 July 2015 / Published online: 17 July 2015

(C) Springer-Verlag Berlin Heidelberg 2015

Keywords Hysteroscopic morcelation · Morcelation . Myoma $\cdot$ Fibroids

\section{Introduction}

Large uterine submucous fibroids can cause severe clinical symptoms, depending on size and location [1]. The standard technique to remove uterine intracavitary abnormalities is resectoscopy [1]. Electroresection is used to remove polyps, type 0 or I myoma $<5 \mathrm{~cm}$ diameter, whereas type II myomas are commonly treated in more than one procedure $[3,6]$.

Hysteroscopic morcelation (HM) is a promising technique to remove myomas. HM reduces tissue by mechanical cutting into small fragments [2-4], whereas subsequent aspiration leads to tissue collection. Several studies indicated successful removal of myomas (type I or 0 ) and polyps by HM [3]. Consistency of myomas can be a significant factor using morcelation. This communication describes the first successful morcelation of a soft 6-cm type II myoma.

Karin Abbink

karinabbink@hotmail.com

Department of Obstetrics and Gynaecology, Catharina Hospital Eindhoven, Michelangelolaan 2, 5623 Eindhoven, The Netherlands

2 Women's Specialized Hospital, King Fahad Medical City Riyadh, Riyadh, Kingdom of Saudi Arabia

3 Maternity Hospital, IVF unit, Sulaiman al Habib Medical Complex, Riyadh, Kingdom of Saudi Arabia

\section{Case}

A woman (39 years) visiting the outpatient Gynaecology $\mathrm{Clinic}^{2}$, complaining of heavy regular menstrual bleeding (HMB), revealed at diagnostic workup a 6$\mathrm{cm}$ submucous uterine fibroid (Fig. 1a, b). Her history included four caesarean sections and a left salpingectomy. Leuprolide acetate showed no benefit. Hysteroscopic removal of the myoma was attempted by using the HM. The patient received misoprostol and antibiotics preoperatively and was counselled for multiple procedures. During an international workshop ${ }^{2}$, complete morcelation using HM (TRUCLEAR; Smith and Nephew, Andover, MA, USA) was performed, using a reciprocating $5-\mathrm{mm}$ blade (Fig. 2). Intrauterine pressure of $100 \mathrm{mmHG}$, a rotary speed of $1400 \mathrm{rpm}$ and a suction of $200 \mathrm{~mm} \mathrm{Hg}$ was used to morcelate the intracavitary part of the myoma. The remaining intramural part was enucleated and morcelated manoeuvring the stiff blade between the myoma and the capsula. The procedure lasted $15.4 \mathrm{~min}$ with 450 cc fluid deficit. Finally, no adherent intracavitary remnants remained (Fig. 1c).

\section{Discussion}

This communication describes a complete hysteroscopic morcelation of a soft 6-cm, type II myoma. Studies using HM describe safe resection of type 0 or I myomas [2, 3, 5]. HM is easy to perform, demonstrates less fluidrelated complications and shows a shorter learning curve, 
Fig. 1 a Pre-operative hysteroscopic view of intracavitary part of the myoma (the arrow points at the surface of the myoma); $\mathbf{b}$ US position of myoma in the uterus; the callipers indicate the outline of the myoma; c post-operative view of the capsule of the enucleated myoma (arrow pointing to capsule, small myoma fragment above); d all material of the myoma as collected in the tissue trap
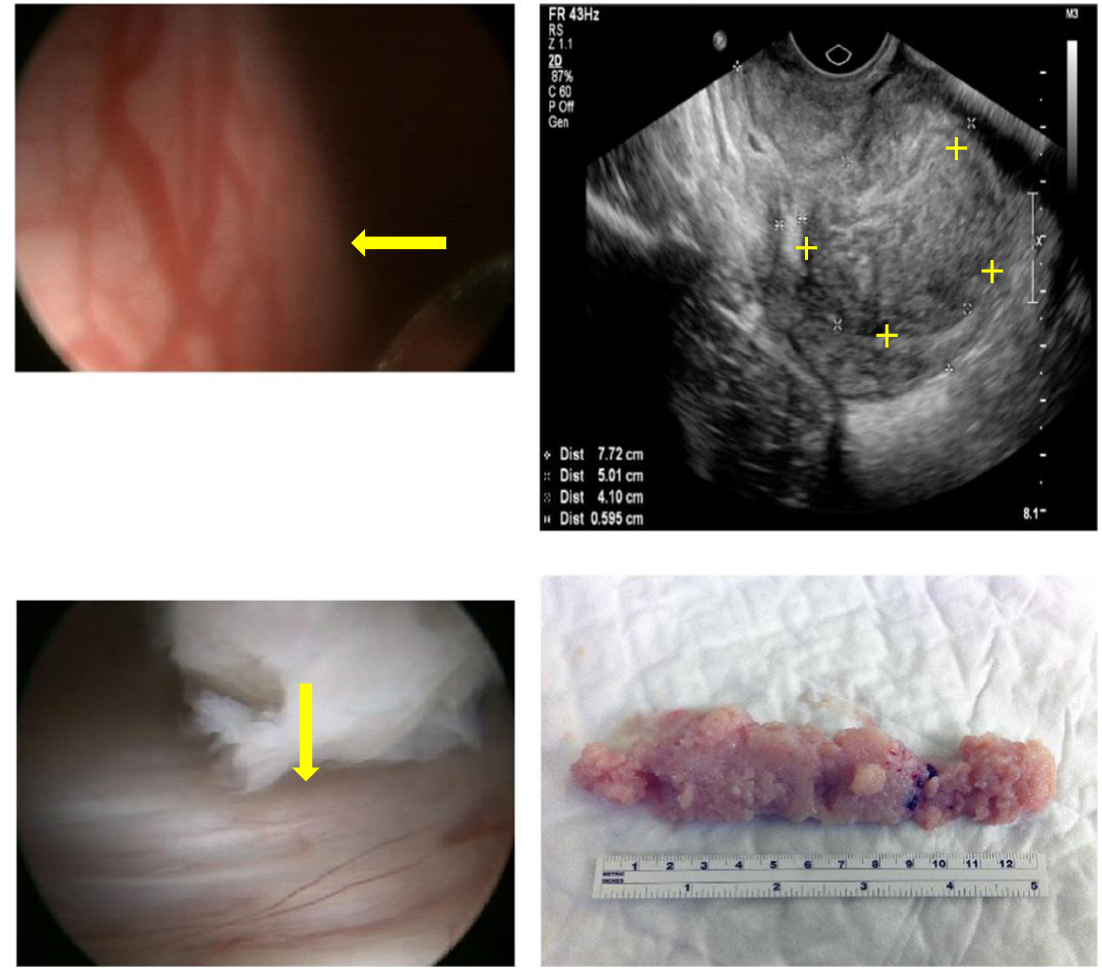

compared to traditional resectoscopes $[2,3,5]$. Significant reduction in operation time may be due to simultaneous aspiration of tissue fragments rather than the removal of each individual fragment by using resectoscopy [1-3]. Risks of complications increase in type II myoma with increased intramural extension [6, 7]. Overall, success rates are lower with higher rates of incomplete resection, post-operative bleeding and reoperation [7].

Probably the soft tissue characteristics led to complete removal in a short operation time, with minimal fluid loss. There is more adaption of tissue into the beak of the device, and larger tissue fragments could be morcelated. The tip of the device is able to dissect the myoma out of its capsula. Despite the absence of electrical current, no excessive bleeding or other complications occurred which demonstrates the future opportunities of HM.

Furthermore, it could be of importance to examine the consistency of the myoma (e.g. using dynamic sonography and elastography) in the pre-operative assessment prior to hysteroscopic removal.

Morcelation of a type II myoma should be performed in select cases to optimize outcome with notice of the consistency of the myoma.
Fig. 2 TRUCLEAR

hysteroscopic morcellator
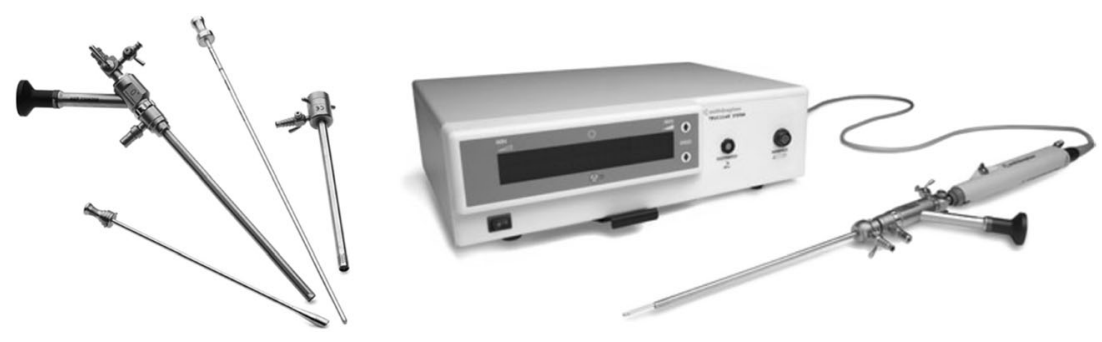
Authors' contribution Karin Abbink wrote the manuscript. Dick B.C. Schoot collected data and edited the manuscript. Sendy Sameer and Tawfig H. Gaafar collected data.

Conflict of interest The authors declare that they have no competing interest.

Informed consent Informed consent was obtained from the participant included in the study.

Ethical approval All procedures performed in studies involving human participants were in accordance with the ethical standards of the institutional and/or national research committee and with the $1964 \mathrm{Hel}-$ sinki Declaration and its later amendments or comparable ethical standards.

\section{References}

1. Di Spiezio Sardo et al (2008) Hysteroscopic myomectomy: a comprehensive review of surgical techniques. Hum Reprod Update 14(2):101-119
2. Emanuel et al (2005) The intra uterine morcellator: a new hysteroscopic operation technique to remove intrauterine polyps and myomas. J Minim Invasive Gynecol 12(1):62-66

3. Hamerlynck et al (2011) Clinical implementation of the hysteroscopic morcellator for removal of intrauterine myomas and polyps. A retrospective descriptive study. Gynecol Surg 8(2):193-196

4. Rubino et al (2015) Twelve-month outcomes for patients undergoing hysteroscopic morcellation of uterine polyps and myomas in an office or ambulatory surgical center. J Minim Invasive Gynecol 22(2): 285-290

5. van Dongen et al (2008) Hysteroscopic morcellator for removal of intrauterine polyps and myomas: a randomized controlled pilot study among residents in training. J Minim Invasive Gynecol 15(4):466471

6. Indman (2006) Hysteroscopic treatment of submucous myomas. Clin Obstet Gynecol 49(4):811-820

7. Wamsteker et al (1993) Transcervical hysteroscopic resection of submucous fibroids for abnormal uterine bleeding: results regarding the degree of intramural extension. Obstet Gynecol 82(5):736-740 